\title{
Control of ultralow pressures: an absolute thermodynamic manometer
}

\author{
Peter Taborek and David Goodstein \\ California Institute of Technology, Pasadena, California 91125 \\ (Received 7 August 1978; in final form, 13 October 1978)
}

A method of accurately manipulating the pressure of helium gas in the range $P=10^{-1}$ to $10^{-20}$ Torr, $T<5 \mathrm{~K}$ is presented. The method requires only a very modest apparatus and makes use of thermodynamic measurements on the ${ }^{4} \mathrm{He}-\mathrm{Grafoil}$ system. A calibration curve and a pressure scale for the manometer are developed which will allow convenient application in other laboratories. An experiment which utilizes the technique is briefly disscussed.

\section{INTRODUCTION}

In the course of presenting a combined thermodynamic analysis of heat capacity and vapor pressure data for the system ${ }^{4} \mathrm{He}$ adsorbed on Grafoil, Elgin and Goodstein ${ }^{1}$ reported that a certain phenomenon, the "ordering peak," occurred at a vapor pressure of $1.4 \times 10^{-17}$ Torr. The datum itself was of no importance except to illustrate the point that arbitrarily low pressures could be measured by their technique. In this paper we should like to show how the technique can be used for manometry in suitable cases. We present data and instructions necessary for use of the technique, and briefly describe an example of an experiment in which it is currently being applied.

The system ${ }^{4} \mathrm{He}$ on Grafoil has been studied extensively because of interest in helium films. The use of Grafoil $^{2}$ in particular as a substrate has had a revolutionary effect on thin film studies because it is cheap, readily available, has an extremely homogeneous surface, and yields experimental results that are reproducible from one laboratory to another and one sample to another to a degree that is probably unprecedented in surface physics. Those same properties which have made the helium-Grafoil system the subject of intense study, ${ }^{3}$ together with the results of those studies also make it suitable and convenient for manometry.

The basic principle of the device is as follows. An adsorbed film in thermodynamic equilibrium has the same temperature $T$ and chemical potential $\mu$ as its vapor. At relatively high temperature and coverage (amount adsorbed) $N$, the vapor pressure $P$ is easily measured, and the chemical potential of the gas, and hence of the film, may be obtained from a standard formula,

$$
\mu=k T \ln \left[\frac{P}{k T}\left(\frac{2 \pi \hbar^{2}}{m k T}\right)^{3 / 2}\right] .
$$

At lower $T$ and $N, P$ becomes difficult to measure, but the heat capacity of the film may still be measured. Integrating the heat capacity with respect to $\ln (T)$ for various values of $N$, one can construct the entropy
$S$, as a function of $N$ and $T$. From these data, changes in $\mu$ may be deduced using the Maxwell relation,

$$
\left(\frac{\partial \mu}{\partial T}\right)_{N}=-\left(\frac{\partial S}{\partial N}\right)_{T} .
$$

These changes in $\mu$ with $T$ may be used to correct the measured high temperature values, thus yielding $\mu$ for all $T$ and $N$. The original procedure is now reversed, the pressure of the gas being deduced from $\mu$ and $T$. In this way one may measure the pressure of the gas, no matter how low, so long as the necessary data for $\mu(N, T)$ are available.

In practice, one prepares an experimental cell in which a convenient quantity of Grafoil is installed. An amount of helium, $N_{0}$, is measured into the cell, which is then brought to equilibrium at temperature $T$. If the surface area of the Grafoil has been calibrated (see below), and the pressure is sufficiently low that the amount adsorbed is essentially equal to $N_{0}$, the pressure may simply be read off an appropriate table of data.

At present, the necessary data (to be presented in convenient form below) are available only for ${ }^{4} \mathrm{He}$. Consequently, the Grafoil manometer must be used at $T \leqslant 4 \mathrm{~K}$. In principle, other adsorbates may be used if the required data become available. Data for ${ }^{3} \mathrm{He}$ will be presented soon, and work on other gases is in progress.

Clearly, the device being described here is not to be used, for example, to detect the dynamic pressure in an ultrahigh vacuum system. Instead it may be used to monitor and control the absolute thermodynamic pressure of a one component gas. In addition to applications like the one described in Sec. II below, it might be used, for example, for precise control of the atmosphere prior to sputtering or evaporation of metallic films.

There are some important restrictions on the use of this technique. For example, the temperature of the Grafoil must be the lowest temperature anywhere in the system. Moreover, the surface area of the Grafoil must be large compared to all other surfaces at the same temperature. On the other hand, if the region of interest is at the same temperature as the manometer, the measure- 


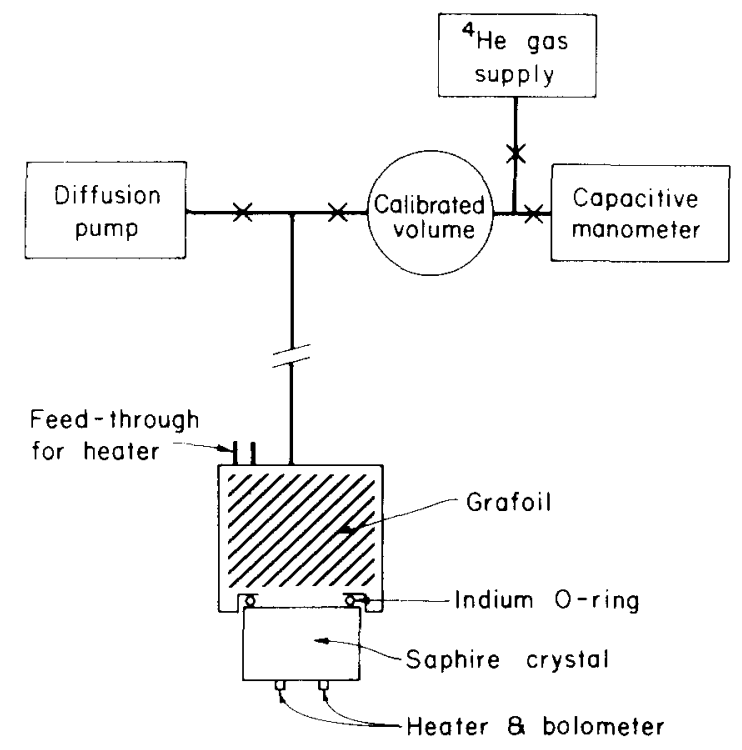

F1G. 1. Schematic diagram of apparatus for heat pulse experiments using the Grafoil manometer. The cell is immersed in a liquid helium bath.

ment is an in situ one, so that there is no thermomolecular correction to be made to the pressure. Finally, care must be taken that the adsorbate has reached its equilibrium distribution in the Grafoil. A dependable technique for assuring equilibrium has been presented by Elgin and Goodstein. ${ }^{1}$

\section{DESCRIPTION OF APPARATUS AND GRAFOIL CALIBRATION}

We are currently conducting experiments designed to study thermal transport at the interface of a dielectric solid and helium using the heat pulse technique. Since anomalously high conductance has been observed at pressures less than $5 \times 10^{-6} \mathrm{Torr},{ }^{4}$ we became interested in developing a method of manometry which would extend to the ultrahigh vacuum regime and which would not suffer from the large thermomolecular corrections required for conventional techniques applied to lowpressure low-temperature measurements.

The apparatus consists of a stainless-steel vacuum can with a sapphire disk attached by means of an indium O-ring seal forming one wall of the can. The sapphire surface in contact with the $\mathrm{He}$ bath has a heater and bolometer for use in the heat pulse experiments. The cell contains a convenient quantity of Grafoil, in our case approximately $6 \mathrm{~g}$, rolled into a spiral and packed sufficiently tightly to make good thermal contact with the stainless steel wall. $91.4 \mathrm{~cm}$ of Evanohm wire was wound through the Grafoil to allow the temperature and pressure to be raised and to facilitate rapid redistribution of the added gas. A 1.6- $\mathrm{mm}$ stainlesssteel gas fill line leads out of the Dewar to a diffusion pump and a gas handling system with a calibrated volume and a capacitive manometer with a range of $10^{-4}$ to $10^{2}$ Torr. A schematic diagram is shown in Fig. 1.

In order to utilize the thermodynamic data on the
He-Grafoil system which has been accumulated at several laboratories, ${ }^{5-8}$ it was necessary to calibrate the adsorption area of our specimen of Grafoil accurately. In previous work, this was accomplished by noting the coverage at which a characteristic peak in the heat capacity was observed. This peak, referred to as the $1 / 3$ ordering peak, is a signature of a configuration in which helium atoms occupy every third hexagon in the graphite lattice. Since the structure and atomic spacing of graphite are well known, this measurement provides a method of determining the adsorption area with an uncertainty of $<0.05 \%$, and allows meaningful comparison of data taken on different samples. The $1 / 3$ ordering coverage provides a natural unit for $N$, the amount of adsorbed gas, which we use to present the calibration curves in a universal manner.

Direct heat capacity measurements are generally not easy to make but it was found that careful comparison of $4.2 \mathrm{~K}$ vapor pressure isotherms was sufficiently accurate to scale the adsorption area to within $1 \%$. Using the combined heat capacity and vapor pressure data of Bretz, Dash, Hickernell, McLean, and Vilches, hereafter referred to as BDHMV,${ }^{5}$ and Elgin, ${ }^{6}$ a $4.2 \mathrm{~K}$ vapor pressure isotherm was constructed and is shown in Fig. 2 in units of $N_{1 / 3}$. The same figure shows points taken on the Grafoil used in this work, in STP $\mathrm{cm}^{3}$ units. The experimental isotherm is constructed by pumping the cell to $10^{-6}$ Torr at room temperature, cooling to $4.2 \mathrm{~K}$, adding measured quantities of gas, and measuring the resulting pressure. $N$ is the amount of material adsorbed, and must be corrected for any residual in the gas phase, particularly at higher pressures. $P$ is the pressure in the cell, obtained from the pressure at room temperature after correcting for the thermomolecular effect $^{9}$ ( $P_{\text {warm }} /$ $P_{\text {cold }}$ may be as high as 15 ). Using Fig. 2 , the $1 / 3$ ordering coverage $N_{1 / 3}$ of any sample of Grafoil can easily be determined. We have checked the accuracy of the tech-

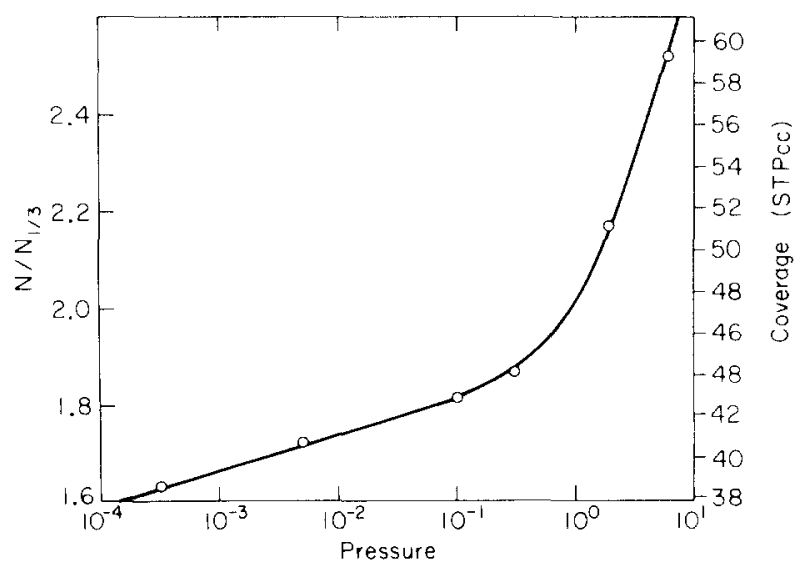

FiG. 2. $4.2 \mathrm{~K}$ vapor pressure isotherm for ${ }^{4} \mathrm{He}$ adsorbed on Grafoil. The solid curve is derived from the same data used to construct Fig. 3, and thus provides a thermodynamically consistent calibration of adsorption area for using the pressure scale shown in Fig. 3. The coverage is shown on the left in units of $N_{1 / 3}$. The circles are measurements on the Grafoil sample used in this investigation, with the coverage given in $S T P \mathrm{~cm}^{3}$ units on the right. By appropriately scaling the coverage, the $1 / 3$ ordering coverage $N_{1 / 3}$ can be determined for any sample of Grafoil. 
nique by deducing the $1 / 3$ ordering coverage from an unpublished $4.2 \mathrm{~K}$ vapor isotherm taken with the BDHMV ${ }^{5}$ cell " $A$ "; the results agree with the value obtained from heat capacity measurements to within $1 \%$.

\section{EVALUATION OF PRESSURE FROM THERMODYNAMIC DATA}

In order to deduce the pressure from the available thermodynamic data, it was necessary to establish the value of the chemical potential $\mu$ in the relevant region of the $N-T$ plane, and then invert Eq. (1). This was accomplished by interpolating the heat capacity data of BDHMV, ${ }^{5}$ Bretz ${ }^{7.8}$ and Elgin $^{6}$ onto a uniform grid, numerically integrating to find the entropy, and then using Eq. (2) to extend the known values of $\mu$ at high temperatures down to $T=0$. Unfortunately, no heat capacity data were available for $N>2.0, T>3.0$, but the pressures in this region are sufficiently high to measure directly and we have done so. Our vapor pressure data and the results of the thermodynamic analysis were used to evaluate $\mu(N, T)$ for $T<5.0, N<2.8$. Table $I$ gives values of $\mu$ in this region on a uniform grid which may be interpolated to reconstruct $\mu(N, T)$. The pressure in Torr is related to the chemical potential $\mu$ in degrees by

$$
P=155.79 T^{2.5} e^{\mu / T} \text {. }
$$

Equation (3) was used to produce the pressure contour plot shown in Fig. 3.

The main features evident in Fig. 3 are explained by the physics of the adsorption process. For cover-

TABLE I. Chemical potential $\mu$ in degrees of ${ }^{4} \mathrm{He}$ adsorbed on Grafoil.

\begin{tabular}{lcrrrrr}
\hline \hline$N / N_{1 / 3}$ & $\mathrm{~T}=0.0$ & $\mathrm{~T}=1.0$ & $\mathrm{~T}=2.0$ & $\mathrm{~T}=3.0$ & $\mathrm{~T}=4.0$ & $\mathrm{~T}=5.0$ \\
\hline 0.0000 & 213.46 & 213.41 & 214.53 & 216.25 & 216.81 & 216.73 \\
0.0936 & 146.52 & 147.39 & 149.36 & 152.63 & 156.46 & 160.47 \\
0.1872 & 145.40 & 146.10 & 147.18 & 148.68 & 150.98 & 153.63 \\
0.2808 & 144.80 & 144.66 & 145.23 & 147.31 & 148.11 & 148.20 \\
0.3744 & 144.60 & 144.52 & 144.39 & 145.63 & 146.14 & 146.23 \\
0.4680 & 143.74 & 143.67 & 143.59 & 144.40 & 145.21 & 145.99 \\
0.5616 & 143.58 & 143.54 & 143.22 & 143.50 & 144.48 & 145.58 \\
0.6551 & 143.47 & 143.28 & 143.04 & 142.85 & 143.50 & 144.21 \\
0.7487 & 143.39 & 143.07 & 142.59 & 141.98 & 141.87 & 142.22 \\
0.8423 & 143.07 & 142.61 & 142.56 & 140.61 & 139.70 & 139.74 \\
0.9359 & 142.47 & 142.31 & 141.06 & 138.36 & 137.19 & 136.86 \\
1.0295 & 134.90 & 134.99 & 135.04 & 134.97 & 134.28 & 133.32 \\
1.1231 & 129.58 & 129.72 & 130.12 & 130.38 & 129.91 & 129.07 \\
1.2167 & 125.38 & 125.09 & 124.94 & 124.73 & 123.97 & 122.94 \\
1.3103 & 119.18 & 118.99 & 118.57 & 117.75 & 116.59 & 115.28 \\
1.4039 & 111.30 & 111.38 & 110.78 & 109.33 & 107.27 & 104.98 \\
1.4975 & 100.15 & 100.07 & 100.02 & 99.18 & 96.47 & 92.86 \\
1.5911 & 86.23 & 86.29 & 86.19 & 85.74 & 84.64 & 83.28 \\
1.6847 & 69.11 & 69.07 & 69.07 & 68.84 & 68.26 & 67.46 \\
1.7783 & 49.39 & 49.53 & 49.36 & 49.42 & 50.03 & 51.09 \\
1.8719 & 33.93 & 33.80 & 33.58 & 34.50 & 38.16 & 43.40 \\
1.9654 & 30.88 & 30.71 & 30.45 & 31.97 & 34.71 & 39.16 \\
2.0590 & 30.27 & 30.18 & 30.10 & 31.36 & 34.61 & 36.36 \\
2.1526 & 29.76 & 29.45 & 29.54 & 30.65 & 33.63 & 34.75 \\
2.2462 & 28.39 & 28.56 & 29.18 & 29.75 & 31.90 & 33.66 \\
2.3398 & 28.27 & 28.40 & 29.03 & 29.32 & 30.89 & 32.43 \\
2.4334 & 28.02 & 28.23 & 28.99 & 29.28 & 30.22 & 31.17 \\
2.5270 & 27.93 & 28.05 & 28.77 & 28.78 & 29.25 & 30.07 \\
2.6206 & 27.09 & 27.60 & 27.97 & 27.92 & 28.23 & 28.96 \\
2.7142 & 23.77 & 24.48 & 25.74 & 27.33 & 27.62 & 27.61 \\
2.8078 & 19.95 & 20.83 & 23.25 & 26.82 & 27.12 & 26.21 \\
\hline \hline & & & & & & \\
& & & & & & \\
\hline
\end{tabular}

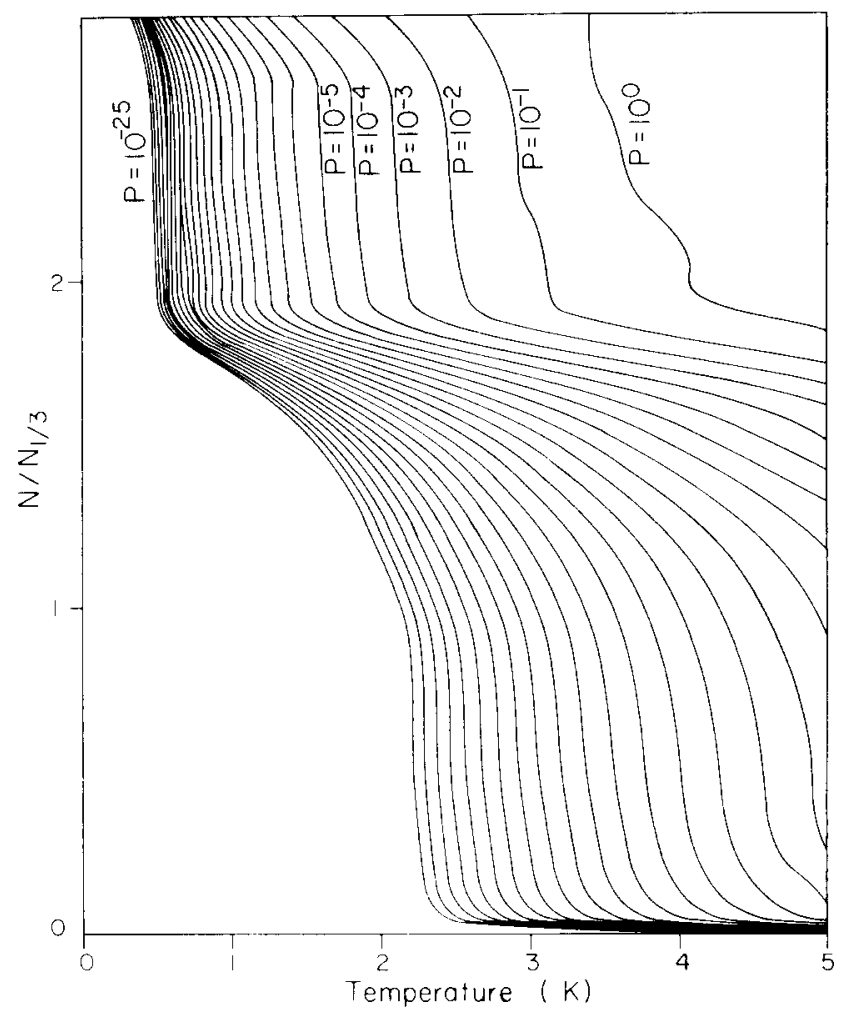

FIg. 3. Logarithmic isobaric contours in units of Torr in the $N-T$ plane for ${ }^{4} \mathrm{He}$ adsorbed on Grafoil.

ages below $N=0.1$, the atoms are preferentially adsorbed at a few sites with high binding energy. Once these sites are occupied, the subsequent atoms see a uniform substrate, and as long as the He atoms are far apart, the binding energy, and thus the pressure, is insensitive to the coverage. As the coverage approaches a complete monolayer, it becomes less energetically advantageous to be in the adsorbed film, and the pressure rises. For $N>2$ the second layer begins on a very uniform substrate of helium-coated graphite with a considerably reduced binding energy. The pressure contains a factor $\exp \left[-E_{\text {binding }} / k T\right]$, so the isobaric contours become very dense at low $T$.

Using Fig. 3, the pressure in a cell containing Grafoil can be varied over a range spanning many orders of magnitude, but the accuracy of the method is affected by several sources of error. In our system, the accuracy of the calibration of the Grafoil area is limited to $\pm 0.5 \%$ by the accuracy of the standard volumes in the gas handling apparatus, and the thermomolecular correction to pressure measurements below 1 Torr. The large thermal gradients which exist in the gas fill line and uncertainties in the cold dead volume contribute to uncertainties in the amount of gas adsorbed, but this effect becomes less important for low pressures and is already negligible for $P<0.1$ Torr. The only contaminant which could possibly influence the results is ${ }^{3} \mathrm{He}$, but because of the low concentrations, the effect is probably minimal. ${ }^{10}$

A basic prerequisite to the use of the thermodynamic data is that the gas and adsorbed film be in equilibrium. 
For pressures in the milliTorr range, equilibrium times are on the order of an hour, but for lower pressures the time required for the material to distribute itself throughout the Grafoil may be as long as weeks. ${ }^{1}$ Elgin and Goodstein found that if the temperature of the cell could be raised until the pressure became sufficiently high to ensure rapid equilibration and then lowered at a rate of $2 \%$ per minute, equilibrium could always be established in a reasonable time. In our apparatus, the heating was accomplished by dissipating less than $0.1 \mathrm{~W}$ of power in the Evanohm wire wound through the Grafoil.

\section{APPLICATIONS OF THE MANOMETER}

An example of the use of this technique is our recent investigation of the effects of film thickness and gas pressure on heat pulse reflection at a helium-sapphire interface. An accurately known quantity of gas, for instance $39.25 \mathrm{STP} \mathrm{cm}^{3}$, is admitted to the experimental cell and forms a film which covers all the surfaces (the few $\mathrm{cm}^{2}$ of sapphire and steel surface are insignificant in the thermodynamic description of the cell which contains nearly $100 \mathrm{~m}^{2}$ of graphite). Using Fig. 2 to scale the area of the Grafoil gives $N=1.66$ in units of $N_{1 / 3}$. Figure 3 shows that $P=1 \times 10^{-3}$ at $4.2 \mathrm{~K}$, and equilibrium takes less than $1 \mathrm{~h}$. Since less than $0.01 \%$ of the helium is in the gas phase, cooling the cell to $1.5 \mathrm{~K}$ reduces the pressure to $P=1 \times 10^{-15}$, but leaves the film thickness essentially unchanged. Following the prescription of lowering the temperature by $2 \% / \mathrm{min}$ to assure equilibrium, this procedure takes about $50 \mathrm{~min}$. By adjusting the coverage and temperature, we have been able to vary the film thickness and gas pressure independently over a wide range with much more accuracy than would be otherwise possible.

Other applications might utilize the ability to measure extremely low pressures. Although the accuracy of the technique is diminished in the low temperature region close to monolayer completion, it is quite feasible to produce pressures as low as $10^{-20}$ Torr with an uncertainty of at most \pm 1 in the logarithm of $P$. Although Fig. 3 could be extended to include even lower pressures, the thermodynamic fluctuations in $P$ become of the order of $P$ for pressures lower than $10^{-20}$ Torr, so these values have little physical significance except for placing upper bounds on the number of atomic collisions per unit area per unit time.

The technique presented above is particularly well suited for manometry at pressures below 1 Torr at low temperatures where other methods are difficult or impossible to use reliably. Since the measurement is based on heat capacity data, there is no need for thermomolecular corrections. The calibration curve of Fig. 2 and the pressure scale of Fig. 3, which can be constructed more accurately using the data in Table I, permit measurements from $10^{-1}$ to $10^{-20}$ Torr with a minimum of care using a very simple apparatus. The accuracy of the method, which is essentially determined by the precision with which the adsorption area can be scaled, depends on the region of the $N-T$ plane in which the measurement is made. A small error in the coverage will not seriously affect pressure measurements for $N>2.0$, but may cause considerable error in the region of monolayer completion.

Although sufficient thermodynamic data to carry out the analysis needed for this application presently exist only for the ${ }^{4} \mathrm{He}$-Grafoil system, other substrateadsorbant combinations might be profitably used for the same purpose. In particular, submonolayer heat capacity data already exist for ${ }^{4} \mathrm{He}-$ Grafoil up to $20 \mathrm{~K}$ and ${ }^{3} \mathrm{He}-\mathrm{Grafoil}$ to $10 \mathrm{~K}$, and work is being done on the $\mathrm{He}-\mathrm{MgO}$ and $\mathrm{N}_{2}$-Grafoil systems.

\section{ACKNOWLEDGMENTS}

We would like to thank O. Vilches for sending us the unpublished vapor pressure isotherm and heat capacity data taken by the Washington group. We also thank M. Bretz for sending us heat capacity data prior to publication, and J. Greif for the use of several computer programs used to analyze the data. The assistance of Sai Wai Fu in taking the vapor pressure data is gratefully acknowledged.

${ }^{1}$ R. L. Elgin and D. L. Goodstein, Phys. Rev. A 9. 2657 (1974)

${ }^{2}$ Grafoil is a form of exfoliated graphite with a specific area of approximately $10 \mathrm{~m}^{2} / \mathrm{g}$. It is commercially available from Union Carbide.

${ }^{3}$ For an introduction to work on films, see J. G. Dash, Films on Solid Surfaces (Academic, New York, 1975).

${ }^{4}$ W. Dietshe and H. Kinder, J. Low Temp. Phys. 23, 27 (1976).

${ }^{5}$ M. Bretz, J. G. Dash, D. C. Hickernell, E. O. McLean, and

O. E. Vilches, Phys. Rev. A 8, 1589 (1973).

${ }^{6}$ R. L. Elgin, Thesis, California Institute of Technology, Pasadena, 1973.

${ }^{7}$ S. E. Polanco and M. Bretz, Phys. Rev. B 17, 151 (1977).

${ }^{8} \mathrm{M}$. Bretz, in Monolayer and Submonolayer Helium Films, edited by J. Daunt and E. Lerner (Plenum, New York, 1973), p. 11.

${ }^{9}$ W. H. Keesom, Helium (Elsevier, Amsterdam, 1942).

${ }^{10}$ The ${ }^{3} \mathrm{He}$ binding energy is a few percent lower than for ${ }^{4} \mathrm{He}$, but at

low coverage, the gas pressure is proportional to N. Since the

${ }^{3} \mathrm{He}$ concentration is typically $7 \mathrm{ppm}$, the effect is negligible. 